\title{
Avoiding permanent atrial fibrillation: treatment approaches to prevent disease progression
}

This article was published in the following Dove Press journal:

Vascular Health and Risk Management

14 December 2013

Number of times this article has been viewed

\author{
Ashish Shukla \\ Anne B Curtis \\ Division of Cardiology, Department \\ of Medicine, State University of \\ New York, Buffalo, NY, USA
}

\begin{abstract}
Atrial fibrillation (AF) is the most common sustained arrhythmia and a major global public health problem due to its associated morbidity, including stroke and heart failure, diminished quality of life, and increased mortality. AF often presents initially in a paroxysmal form and may progress to a more sustained form over time. Evidence from randomized controlled trials suggests that there may be no mortality benefit to using a rhythm control approach in comparison with rate control of AF. Nevertheless, sustained forms of AF may be associated with increased symptoms and cardiovascular morbidity, and consequently they remain an additional target for therapy. The present review evaluates the clinical correlates of arrhythmia progression and risk stratification techniques available to assess probability of AF progression. Further, currently available management options for arrhythmia control in AF are evaluated and their therapeutic effect and efficacy on disease progression are explored.
\end{abstract}

Keywords: atrial fibrillation, progression, permanent, prevention

\section{Introduction}

First described in 1909, atrial fibrillation (AF) is the most common sustained arrhythmia, affecting about 2.7 to 6.1 million people in the USA. ${ }^{1-3}$ It is a significant public health problem globally due to its associated morbidity, mortality, and health-related expenditures. Systematic approaches to reducing the burden of AF have been studied extensively, from investigation of its clinical epidemiology, evaluation of risk factors that promote its onset and progression from a paroxysmal to a more sustained form, and evaluation of therapeutic options to reduce the morbidity and mortality associated with this arrhythmia.

In this review, we address the current knowledge about the epidemiology of AF, along with its natural history, clinical course, and progression. We further seek to summarize the currently available therapeutic options as well as evaluate factors that might have the potential to affect progression of AF. A better understanding of the multiple factors that influence this condition is increasingly important in order to reduce the impact of this arrhythmia on diverse patient populations.

\section{Incidence and prevalence of AF}

The prevalence of AF in the USA is projected to rise to between 5.6 million and 12 million by the year 2050.,3 Epidemiological studies from Western Europe report a similarly high prevalence, ranging from $2.0 \%$ to $4.7 \%$ of the population. ${ }^{4,5}$ Outside of North America and Europe, the data on the prevalence of AF are less robust. ${ }^{6}$ The correlation of increasing patient age with increasing prevalence of AF has been reported
Condence: Anne B Curtis and Chair, Department of Medicine University at Buffalo, 100 High Street, D2-76, Buffalo, NY 14203, USA

Email abcurtis@buffalo.edu 
in the Framingham study, the Cardiovascular Health study, as well as other studies conducted in Australia, Holland, and Scotland. ${ }^{7}$ Based on the results from multiple communitybased cohort studies conducted in the USA and Europe, the lifetime risk of AF is approximately $22 \%-26 \%$ in men and $22 \%-23 \%$ in women by 80 years of age. ${ }^{8,9}$ The risk of AF increases from $1 \%$ in individuals aged $50-59$ years to $10 \%$ between the ages of 80 and 84 years, and subsequently to $11 \%-18 \%$ beyond 85 years of age. ${ }^{2,3}$

Most of the evidence regarding the prevalence of AF has been derived from studies that enrolled symptomatic patients with electrocardiographic documentation of the arrhythmia. Since AF often starts in a paroxysmal form and many patients can have asymptomatic AF, it appears likely that the true prevalence of AF may indeed be higher than previously reported. The Asymptomatic Atrial Fibrillation and Stroke Evaluation in Pacemaker Patients and the Atrial Fibrillation Reduction Atrial Pacing Trial (ASSERT) monitored patients 65 years or older who had hypertension and no prior history of AF and a newly implanted pacemaker or cardioverter defibrillator for development of subclinical atrial tachyarrhythmias (defined as episodes of atrial rate $>190$ beats per minute for more than 6 minutes). At 3 months, $10.1 \%$ of the enrolled patients had developed such episodes. Subclinical AF was indeed associated with both an increased risk of clinical AF and ischemic stroke and systemic embolization. ${ }^{10}$ Similar results have been reported from earlier studies that employed continuous cardiac monitoring devices. These studies have estimated AF to be present in $23 \%$ of patients presenting with cryptogenic transient ischemic attack, further indicating that there is a higher burden of AF and its consequent morbidity and mortality than has been assumed. ${ }^{11,12}$

\section{Classification of AF}

The current classification system for AF is based on a consensus to maintain simplicity and clinical relevance. It recognizes a few major stages in the spectrum of AF. The 'first detected' AF episode represents the initial detection of AF in a patient by a health care provider. This often requires further description as to the duration of the arrhythmia and whether or not it was symptomatic. AF becomes 'recurrent' when a patient has two or more episodes. Recurrent AF is either labeled 'paroxysmal' when it is self-terminating or 'persistent' when it lasts more than 7 days or is terminated earlier using either pharmacological means or direct current cardioversion. For AF that has persisted for over a year, if a rhythm control strategy is pursued, the arrhythmia is categorized as 'long-standing persistent AF.' On the other hand, if the patient is in AF and attempts to maintain sinus rhythm have been abandoned, either because of futility or the preference of the patient and the physician, the AF is labeled 'permanent." ${ }^{13}$

'Lone AF' and 'non-valvular AF' describe the patient's clinical milieu under which AF has developed. Lone AF is the term used if it develops in individuals under 60 years of age without any evidence of either cardiac or pulmonary disease, including hypertension. This category has been developed primarily to indicate the overall favorable prognosis with respect to thromboembolism and mortality. Designation of non-valvular AF, the subject of this review, is restricted to those patients who develop AF in the absence of valvular heart disease.

\section{Risk factors, cardiovascular remodeling, and primary prevention of AF}

In addition to increasing age, several other comorbidities such as male sex, heart failure, hypertension, diabetes mellitus, obesity, cigarette smoking, and myocardial infarction have individually been identified as factors that promote development of AF. Subclinical markers such as structural heart disease (left atrial enlargement, left ventricular hypertrophy), inflammation, neurohormones, obstructive sleep apnea, and metabolic syndrome are additional factors that contribute to the development of AF.

In addition to clinical comorbidities, structural changes and electrophysiological remodeling have been identified as important contributors to the development of AF. Atrial fibrosis, often a sequel to atrial injury and inflammation, is known to have a strong association with the development and persistence of AF in both animal models and human studies. A large number of cytokines, signaling pathways including transforming growth factor- $\beta$, platelet-derived growth factor, and the renin angiotensin-aldosterone system (RAAS) have been implicated in the development of atrial fibrosis. Given these observations, prevention of atrial inflammation and fibrosis has been hypothesized to be a major target in prevention of AF. ${ }^{14}$

Development of AF is also known to be associated with altered electrical activation and remodeling of the atrium. Increased left atrial pressure and dilatation lead to alterations in the expression and function of ion channels. The resulting ectopic triggers and micro-reentrant circuits in the atrium and pulmonary veins facilitate initiation and maintenance of AF.

Data from observational studies indicate that lifestyle changes, diet, and the use of certain medications may be 
associated with lower AF rates. Clinical studies in patients with heart failure, hypertension, and myocardial infarction have suggested that the use of RAAS inhibitors may be associated with a $28 \%$ reduction in new onset AF. Similarly, the Atorvastatin for Reduction of Myocardial Dysrhythmia After Cardiac Surgery (ARMYDA-3) trial found that initiation of a statin after elective cardiac surgery reduced in-hospital AF to a rate of $35 \%$ in comparison with a rate of $57 \%$ in patients who received placebo. However, other clinical studies that have explored the use of RAAS inhibitors or statins (due to their anti-inflammatory and other effects) have shown mixed results in preventing AF.

\section{Progression of AF and its predictors}

The natural history of AF and its progression from selfterminating paroxysms to a more sustained or permanent state has been evaluated in multiple observational studies including data registries. The number and severity of underlying patient comorbidities heavily influence this transition, with the reported rates of progression to persistent AF ranging from $9 \%$ to up to $30 \%$ at 1 year. ${ }^{15-18}$ The Euro Heart survey on AF reported that $15 \%$ of patients with AF progressed to persistent AF in 1 year. In the Japanese Rhythm Management Trial for Atrial Fibrillation (J-RHYTHM II), the progression rate was approximately $10 \%$ per year in patients with hypertension and paroxysmal AF. ${ }^{19}$ In the Canadian Registry of Atrial Fibrillation (CARAF) study, the probability of recurrence of AF over 5 years in patients enrolled with a first documented AF episode was $63 \%$, and, overall, $25 \%$ of patients progressed to permanent AF within 5 years. The time to progression of $\mathrm{AF}$ is reported to be longer from a first reported episode to recurrent $\mathrm{AF}$ in comparison with the time from persistent to a permanent form, suggesting an acceleration in the rate of progression with increasing arrhythmia burden. ${ }^{20}$

A majority of patients with new-onset AF, except for those associated with a postoperative state or a transient acute illness, are likely to experience recurrences of AF. Among the factors that promote recurrence and progression, multiple studies have reported an overlap between those that promote progression of $\mathrm{AF}$ and factors that are related to the development of new-onset AF. Increasing age, underlying cardiomyopathy, hypertension, AF duration $>3$ months, adoption of a rate-control rather than a rhythm-control strategy, left atrial dilatation, and obesity are all well recognized factors associated with progression of AF from a paroxysmal to a more sustained form. ${ }^{21}$ In the Registry on Cardiac Rhythm Disorders Assessing the Control of AF (RECORD AF) registry, persistent AF at baseline, AF duration $>3$ months, patient age $>75$ years, heart failure, and a rate-control strategy all predicted progression to permanent $\mathrm{AF}^{22} \mathrm{~A}$ few other factors, such as significant aortic stenosis or mitral regurgitation and a slower heart rate at initial presentation with $\mathrm{AF}$, have also been reported in some studies to promote progression of AF, suggesting an interplay of structural remodeling of the heart with chronic stretch and atrial dilatation in addition to systemic metabolic and inflammatory factors. The HATCH scoring system (based on underlying hypertension, age $>75$ years, history of transient ischemic attack or stroke, chronic obstructive pulmonary disease, and heart failure) was proposed to predict this risk of progression in patients receiving pharmacological therapy. ${ }^{16}$ (Using this model, presence of heart failure and transient ischemic attack/stroke are awarded 2 points each while the remaining risk factors are awarded 1 point each.) In this study, AF progressed in $50 \%$ of patients with a HATCH score of $>5$ compared with only $6 \%$ in patients with a score of 0 (Figure 1). The HATCH scoring system has increasingly been found to be useful, although the value of this scoring system has been reported to be confined to patients treated by pharmacological means. In patients who have undergone catheter ablation for AF, this scoring system has not been reported to predict progression of $\mathrm{AF}^{23}$

The clinical outcomes of patients who exhibit progression of AF are worse with respect to hospital admissions and major adverse cardiovascular events. In the Euro Heart survey, patients with AF that had progressed to persistent or permanent AF had higher rates of hospital admissions, transient ischemic attack, stroke, myocardial infarction, and death. ${ }^{16}$ Additionally, permanent AF may be associated with more severe symptoms and lower quality of life.

\section{Therapeutic options in AF Rate control for AF}

Rate-control therapy for AF is pursued with the intention of reducing symptoms and improving cardiovascular hemodynamics by allowing increased time for ventricular filling. As a treatment strategy, there is no specific effort to maintain sinus rhythm and thus no expectation that progression of $\mathrm{AF}$ will be prevented. Reasons for pursuing this strategy include circumventing the adverse effects of antiarrhythmic drugs and avoiding repeated cardioversions. Drugs used for rate control include beta-adrenergic blockers, nondihydropyridine calcium channel antagonists, and dioxin. Amiodarone is occasionally used for this purpose in elderly patients with paroxysmal $\mathrm{AF}$, although it is best avoided in permanent $\mathrm{AF}$ given its potential toxicity. 


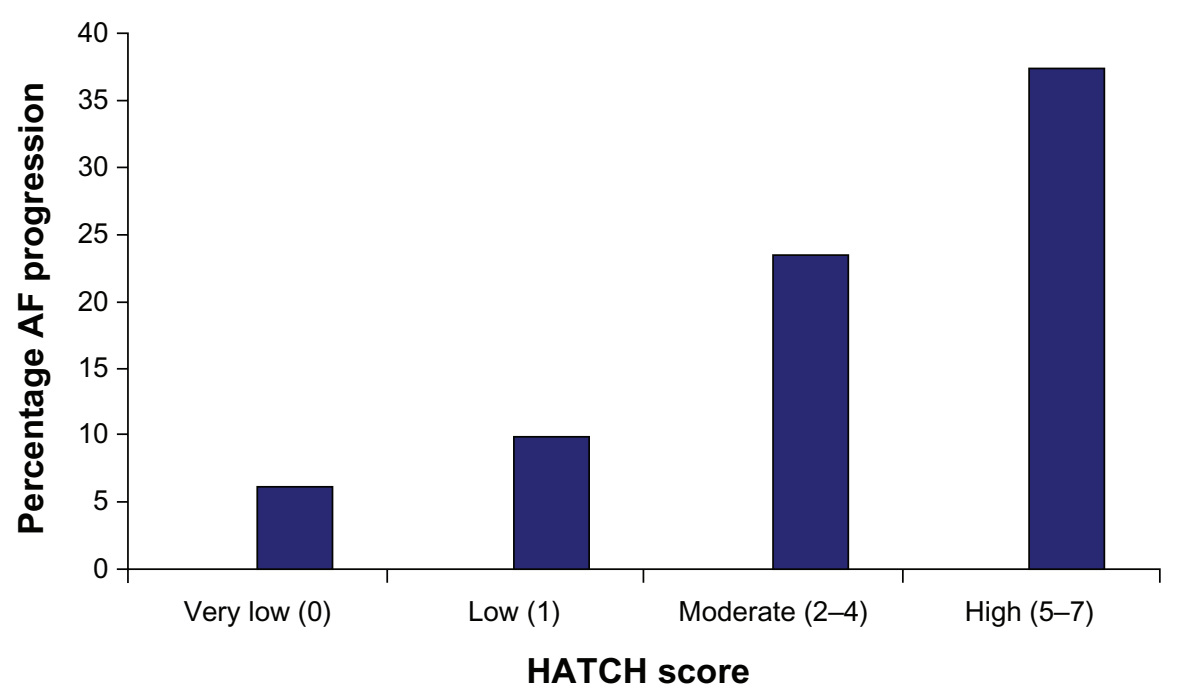

Figure I I-year incidence of progression of atrial fibrillation based on HATCH score (hypertension, age $>75$ years, transient ischemic attack or stroke, chronic obstructive pulmonary disease, and heart failure). ${ }^{16}$

Note: Reprinted from Journal of the American College of Cardiology, 55(8), de Vos CB, Pisters R, Nieuwlaat R, et al, Progression from paroxysmal to persistent atrial fibrillation clinical correlates and prognosis, 725-73I, Copyright (C) 2010, with permission from Elsevier.

Abbreviation: $A F$, atrial fibrillation.

The parameters for optimal rate control have been controversial, with prior studies recommending ventricular rates of $80-100$ beats per minute at rest and $90-110$ beats per minute during moderate exercise based on the results from the Atrial Fibrillation Follow-up Investigation of Rhythm Management (AFFIRM) trial. ${ }^{24}$ However, based upon the results of the Rate Control Efficacy in Permanent Atrial Fibrillation: a Comparison between Lenient versus Strict Rate Control II (RACE II) study, the American College of Cardiology (ACC)/ American Heart Association (AHA)/Heart Rhythm Society (HRS) guidelines have moderated their stance with respect to the value of a strict rate-control strategy. ${ }^{25}$ Thus, in patients with persistent AF with no significant symptoms and stable left ventricular function, a target of maintaining a resting heart rate of $<110$ beats per minute may be appropriate. ${ }^{26}$

\section{Strategies to achieve rhythm control}

In patients with recurrent $\mathrm{AF}$, a decision needs to be made as to whether to pursue a rate- or a rhythm-control strategy. Even so, the two strategies are somewhat inter-related, as patients on rhythm control are usually also prescribed ratecontrol agents. The currently available options for rhythm control include the use of antiarrhythmic drug therapy and catheter ablation of AF. The main goal of rhythm control is to provide symptom relief with reduction in the frequency and duration of the arrhythmia. Less well established reasons for pursuing maintenance of sinus rhythm include improvement in cardiac function and reduction of adverse mechanical and electrical remodeling of the atria.
Cardioversion, either by electrical or by pharmacological means, can be a useful tool once a decision has been made for a rhythm-control strategy in a patient with AF. Cardioversion by itself will not prevent progression of AF to a permanent form, although it can be especially helpful in situations where there has been correction of the circumstances that led to AF. Especially for first episodes of AF, cardioversion may leave the patient free of recurrence for months or longer, especially in the absence of a well developed myocardial substrate for AF maintenance.

\section{Antiarrhythmic drug therapy}

Antiarrhythmic drugs are the first line of therapy for maintenance of sinus rhythm in patients with AF. The majority of these drugs act by reducing the likelihood of reentry by prolonging the atrial effective refractory period through the inhibition of $\mathrm{K}^{+}$currents or reduction of atrial excitability via inhibition of $\mathrm{Na}^{+}$currents. However, most of these drugs affect multiple other ion channels as well as adrenergic receptors. Drugs that affect multiple channels are more effective for maintenance of sinus rhythm than selective ion channel blockers. Stabilization of $\mathrm{Ca}^{2+}$ handling abnormalities and normalization of gap junction physiology have been other targets for treatment of $\mathrm{AF}^{27,28}$

Figure 2 shows the antiarrhythmic drugs that are currently available, along with a proposed algorithm to maintain sinus rhythm. Each of these drugs varies in its efficacy for maintaining sinus rhythm and possesses unique adverse effect profiles. Disopyramide and quinidine, class IA agents that are effective in $\mathrm{AF}$, have fallen out of favor due to their adverse effect 


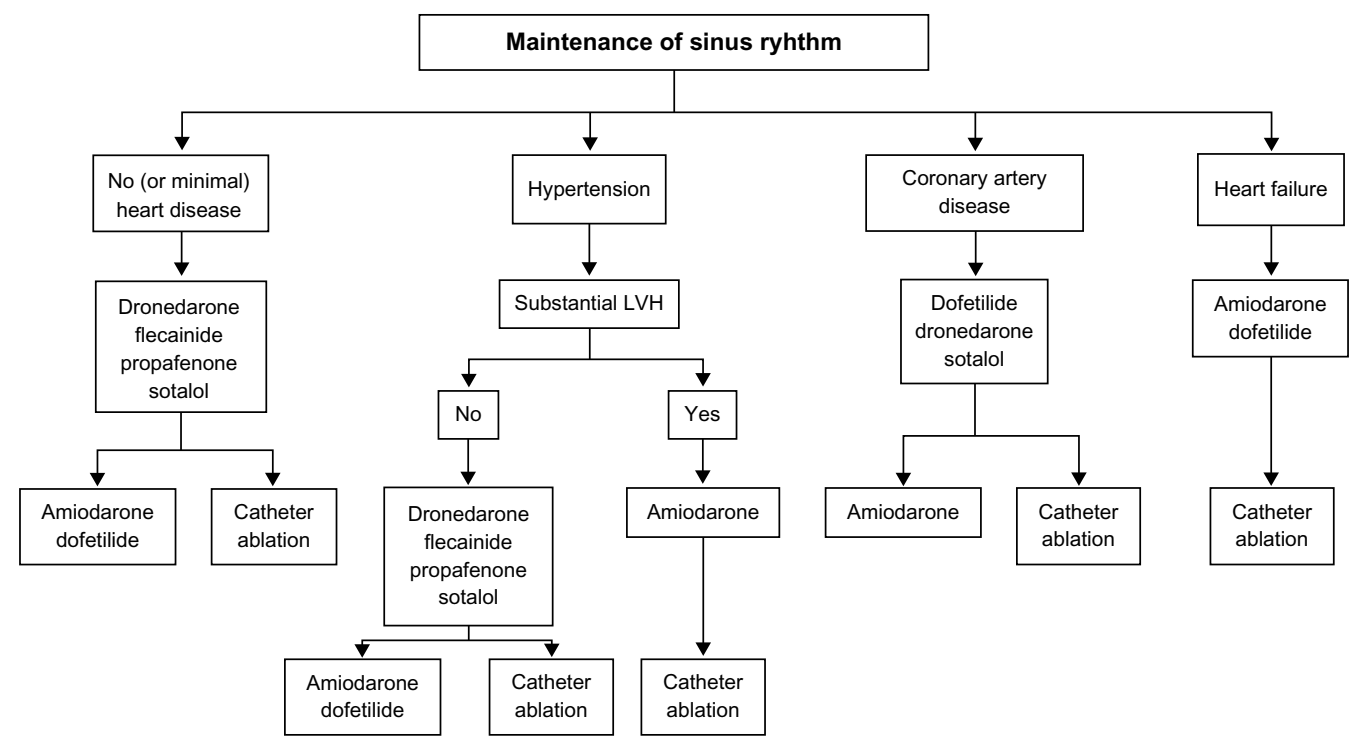

Figure 2 Proposed algorithm for use of antiarrhythmic drugs in patients with paroxysmal or persistent atrial fibrillation. ${ }^{13}$

Note: Reproduced from Fuster V, Rydén LE, Cannom DS, et al, 201I ACCF/AHA/HRS focused updates incorporated into the ACC/AHA/ESC 2006 guidelines for the management of patients with atrial fibrillation: a report of the American College of Cardiology Foundation/American Heart Association Task Force on practice guidelines, Circulation, 123(10):e269-e367, Copyright @ 20I I, with permission from Wolters Kluwer Health.

Abbreviation: LVH, left ventricular hypertrophy.

profiles, including worsening of heart failure and increased mortality. ${ }^{29-33}$ Due to its anticholinergic activity, long-acting disopyramide does have a role in vagally mediated $\mathrm{AF} \cdot{ }^{13}$

Flecainide and propafenone are Class IC arrhythmic agents recommended for the management of $\mathrm{AF}$ in patients without structural heart disease. When compared with placebo, both are effective for maintenance of sinus rhythm and for prolongation of the time to recurrence of $\mathrm{AF}^{34,35}$ Since both of these drugs may have a propensity to promote 1:1 atrioventricular (AV) conduction during atrial flutter, an AV nodal blocking agent is routinely co-administered. In addition to its $\mathrm{Na}^{+}$channel-blocking effect, propafenone has some additional beta-adrenergic-blocking effects.

Class III drugs used to maintain sinus rhythm include amiodarone, dronedarone, sotalol, and dofetilide. Amiodarone is not approved by the US Food and Drug Administration for rhythm control of AF; however, it is one of the most commonly prescribed antiarrhythmic drugs for this condition. In addition to inhibition of the outward potassium currents (Class III effect), amiodarone also has class I $\left(\mathrm{Na}^{+}\right.$channel blocking), Class II (antiadrenergic), and Class IV $\left(\mathrm{Ca}^{2+}\right.$ channel blocking) effects. From the efficacy standpoint, amiodarone is the most potent antiarrhythmic drug available to maintain sinus rhythm and prolong recurrence of AF. Limited data are available to directly compare its efficacy with other antiarrhythmic drugs, although studies that compared it with sotalol and propafenone found amiodarone to be superior. ${ }^{36-38}$ In turn, systematic reviews and indirect comparison meta-analyses have suggested the superiority of amiodarone over other available antiarrhythmic drugs for maintenance of sinus rhythm. ${ }^{39,40}$ The use of amiodarone may be limited by significant cardiovascular and non-cardiovascular adverse effects. The use of amiodarone requires surveillance for lung, liver, and thyroid toxicity, which involves evaluation at baseline and then follow-up evaluations every 6 months or yearly. ${ }^{41}$

Dronedarone is a non-iodinated congener of amiodarone developed with the hypothesis that deletion of the iodine moiety would lead to fewer adverse effects while retaining the antiarrhythmic properties of amiodarone. Randomized trials evaluating dronedarone reported its efficacy in maintaining sinus rhythm, reduction in hospitalization and cardiovascular mortality ${ }^{42,43}$ However, in patients with advanced heart failure, its use was associated with increased mortality. In the Permanent Atrial Fibrillation Outcome Study Using Dronedarone on Top of Standard Therapy (PALLAS) study, which studied patients with permanent $\mathrm{AF}$, dronedarone was associated with an increased risk of stroke, cardiovascular death, and hospitalizations. ${ }^{44,45}$ Dronedarone is now considered reasonable to reduce hospitalization for cardiovascular events in patients with paroxysmal AF. Its use should be reserved for selected low-risk individuals who may have failed other antiarrhythmic drugs. ${ }^{25,46}$

Sotalol is a $\mathrm{K}^{+}$ion channel blocker effective at preventing recurrences of $\mathrm{AF}$ in comparison with placebo at doses ranging from 80 to $160 \mathrm{mg}$ twice daily. In the Canadian Trial of Atrial Fibrillation (CTAF) study, sotalol and propafenone 
appeared to be equally efficacious, while amiodarone had better efficacy. However, upon consideration of the number of patients who discontinued amiodarone due to adverse effects, the difference between the efficacy of amiodarone and sotalol was less marked. ${ }^{36}$ In addition to its antiarrhythmic activity, sotalol also has non-selective beta-adrenergic-blocking properties and is known to provide efficient rate control in cases of AF recurrence. ${ }^{47}$ Sotalol may cause bradycardia and proarrhythmia due to QT prolongation. Thus, it is usually recommended that patients be hospitalized for close cardiac rhythm monitoring upon initiation of the drug as well as with each upward dose adjustment.

Dofetilide, another Class III antiarrhythmic agent, was studied in the Symptomatic Atrial Fibrillation Investigative Research on Dofetilide (SAFIRE-D) study, which reported a $58 \%$ efficacy in maintaining sinus rhythm at 1 year with dofetilide in comparison with $25 \%$ in the placebo group. ${ }^{48}$ In the Danish Investigations of Arrhythmia and Mortality on Dofetilide (DIAMOND) study involving patients with reduced left ventricular systolic function, the dofetilide group had a $79 \%$ probability of maintaining sinus rhythm in comparison with $42 \%$ with placebo at 1 year in addition to a reduced risk of all-cause or congestive heart failure-related hospitalization. ${ }^{49}$ In this study, torsades de pointes occurred in $1.6 \%$ patients, and half of those occurred on day 2 of dofetilide treatment. Due to this risk of torsades, initiation of this drug requires a mandatory inpatient loading period for 3 days with titration of the dose based upon QT interval and renal function. Ibutilide, another class III antiarrhythmic available in an intravenous form, is used mostly for acute conversion to sinus rhythm and is not used as maintenance therapy to prevent AF recurrence. ${ }^{50-52}$

The intravenous formulation of vernakalant has recently been approved in Europe for pharmacological cardioversion of AF of $\leq 7$ days' onset, or $\leq 3$ days for patients after cardiac surgery. It increases atrial refractoriness and causes rate dependent slowing of atrial conduction through its effects on potassium currents $\left(\mathrm{I}_{\text {to }}, \mathrm{I}_{\text {Ach }}, \mathrm{I}_{\mathrm{Kur}}\right)$ and late cardiac sodium current $\left(\mathrm{I}_{\mathrm{Na}}\right) .{ }^{53}$

\section{Catheter ablation for AF}

Electrical isolation of the pulmonary veins by delivering point-by-point radiofrequency lesions around the ostia of the pulmonary veins forms the mainstay of most AF ablation procedures. Pulmonary vein isolation is the major target in patients with paroxysmal AF, while in patients with non-paroxysmal AF, the targets of ablation continue to be debated and may include creating linear ablation lesions and targeting areas of the left atrium that demonstrate fractionated electrograms during AF (complex atrial fractionated electrograms [CAFEs]). ${ }^{13}$ Several factors determine the success of ablation of AF, including the type of AF (paroxysmal, persistent, or long-standing persistent), left atrial size, left ventricular function, and patient comorbidities such as obesity and sleep apnea. Reported rates of recurrence also depend upon the duration of follow-up of these patients. ${ }^{54}$

Based on the results obtained from several randomized controlled trials that have compared the outcomes of AF ablation with antiarrhythmic drug therapy, catheter ablation for $\mathrm{AF}$ is more effective in maintaining sinus rhythm. ${ }^{55-57}$ A meta-analysis that included four such trials reported a 3.7fold increased probability for arrhythmia-free survival with catheter ablation compared with the use of antiarrhythmic drug therapy. ${ }^{58}$ In another systematic review that included 63 ablation studies, the single procedure success rate of ablation without the use of antiarrhythmic drug therapy was $57 \%$ (95\% confidence interval $[\mathrm{CI}] 50-64)$, multiple procedure success without the use of antiarrhythmic drug therapy was $71 \%$ (95\% CI 65-77), and the multiple procedure success rate with the addition of antiarrhythmic drug therapy was $77 \%$ (95\% CI 73-81). In comparison, this study reported a success rate of $52 \%$ (95\% CI 47-57) with the use of antiarrhythmic drug therapy alone. An important caveat that needs to be recognized when evaluating the results of these trials is that the majority of these studies with catheter ablation enrolled predominantly middle-aged white men with paroxysmal AF and few comorbidities. Additionally, the results from the recent Medical Antiarrhythmic Treatment or Radiofrequency Ablation in Paroxysmal Atrial Fibrillation (MANTRA-PAF) trial indicate that, in patients aged 70 years or less, with a left ventricular ejection fraction over $40 \%$, radiofrequency ablation of AF as a first-line strategy may be as safe and effective as antiarrhythmic drug therapy for maintenance of sinus rhythm. ${ }^{59}$ Although these results appear promising, more long-term data about efficacy and adverse effects are needed before definitive conclusions can be drawn. Based on the ACC/AHA/HRS guidelines, catheter ablation for AF is currently indicated in patients with symptomatic, paroxysmal AF in whom one or more antiarrhythmic drug has failed and who have normal or mildly dilated left atrial size, normal or mildly reduced left ventricular function with no severe pulmonary disease (Class I). Ablation is a Class IIa indication in those with symptomatic persistent AF, while it is a Class IIb indication in those with symptomatic, paroxysmal AF with reduced left ventricular systolic function or with significant dilatation of the left atrium. ${ }^{13}$ 


\section{Strategies to prevent progression of AF}

The presence of sustained AF is known to be associated with increased morbidity and mortality, and attempts to curb AF progression have centered around modulation of factors that are known to promote this development in addition to modulation of the arrhythmia itself. ${ }^{16}$ Comorbidities such as heart failure and hypertension have consistently been reported to be associated with AF progression, but studies directly evaluating interventions to control these comorbidities and their effect on AF progression are limited. The effect of obesity as a factor determining progression to sustained AF has been controversial. ${ }^{17,21,60}$ Increasing body mass index is associated with graded increases in left atrial size, and weight reduction is associated with a reduction in left atrial size, suggesting that reduction in body mass index could be a target for prevention of AF progression. ${ }^{13,61}$ Similarly, the presence of obstructive sleep apnea portends a $25 \%$ increased risk for AF recurrence after catheter ablation, while its treatment is associated with an 8-fold improvement in the success of the procedure. ${ }^{62,63}$ Thus, although the extent of benefit that may be obtained from better control of such individual comorbidities in preventing progression of $\mathrm{AF}$ are less evident, this goal remains an important target in the management of AF.

\section{Effect of rhythm control using antiarrhythmic drug therapy on progression of AF}

In comparison with a rate-control strategy, a rhythm-control approach to AF management is reported to delay progression to a sustained form. ${ }^{17}$ In the RECORD AF registry of patients with recently diagnosed $\mathrm{AF}, 54 \%$ of patients in the ratecontrol arm progressed to permanent AF in comparison with $13 \%$ in the rhythm-control group. Upon application of propensity scoring to account for patient comorbidities, the impact of treatment strategy with rhythm control was found to be favorable (odds ratio [OR] $0.20,95 \%$ CI $0.17-0.25$; $P<0.0001) .{ }^{64}$ In another prospective survey that evaluated patients worldwide, patients treated with rhythm control showed less progression of $\mathrm{AF}$ in comparison with a ratecontrol strategy ( $11 \%$ versus $26 \% ; P<0.001)$. In multivariate logistic regression, rate control rather than rhythm control was an independent predictor of AF progression (OR 3.2, 95\% CI 2.5-4.1; $P<0.0001) .{ }^{17}$ In this study, patients who received class IC antiarrhythmic drugs were found to have less AF progression than those who received other pharmacological therapies (Figure 3). As shown in animal studies, prevention of electrical remodeling of the atrium that occurs with increasing AF burden has been hypothesized to be responsible for delay in AF progression. ${ }^{65}$ Additionally, patients with AF maintained in sinus rhythm are known to have a reduction in left atrial size and improvement of left ventricular systolic function, both of which are important factors associated with AF progression. ${ }^{66}$

It has been argued that a rhythm-control strategy could appear to be more favorable due to its selective application in younger patients with fewer comorbidities, factors that are independently associated with AF progression. However, upon using propensity scoring models to correct for the influence

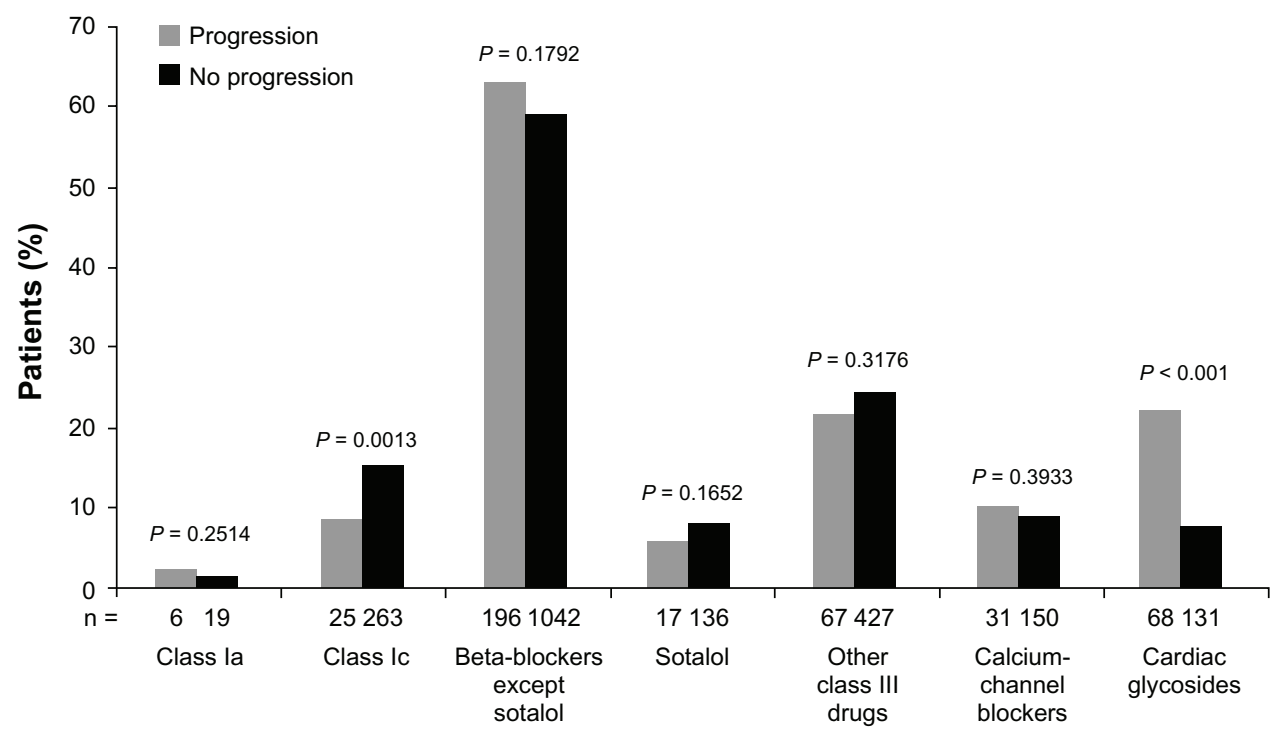

Figure 3 Atrial fibrillation-related medication use at baseline and association with arrhythmia progression at I year. ${ }^{17}$

Note: Reprinted from American Heart Journal, I63(5), De Vos CB, Breithardt G, Camm AJ, et al, Progression of atrial fibrillation in the REgistry on Cardiac rhythm disORDers assessing the control of Atrial Fibrillation cohort: clinical correlates and the effect of rhythm-control therapy, 887-893, Copyright @ 20I2, with permission from Elsevier. 
of these variables, the overall results in the above-mentioned analyses remained unchanged, and a rhythm-control strategy remained a significant deterrent for AF progression.

Use of antiarrhythmic drug therapy is often hampered by limited efficacy in controlling AF over a prolonged duration of time, coupled with an increased risk for adverse effects. In a systematic review evaluating the efficacy outcomes of all antiarrhythmic drugs, the rate of success in control of AF (during the follow-up periods of the included studies) was 52\% (95\% CI 47-57), and drug discontinuation due to adverse effects was $10.4 \%$, along with a $2.8 \%$ overall mortality. ${ }^{67}$ Additionally, in the Euro Heart Survey on AF that examined the natural progression of AF, antiarrhythmic drugs were used in $50 \%$ of patients and amiodarone in approximately $25 \%$, yet the use of these agents was not significantly associated with a reduction in AF progression. ${ }^{16} \mathrm{~A}$ limitation of this study was that patients were not randomized to a specific treatment strategy, and treatment decisions were left to attending cardiologists.

\section{Comparison of catheter ablation with antiarrhythmic drug therapy}

Radiofrequency catheter ablation (RFA) of AF when compared with the use of antiarrhythmic drug therapy has been reported to have more favorable outcomes for reducing the progression of AF. In a study by Pappone et al, ${ }^{20} 106$ patients who presented to the emergency room with a first diagnosed episode of AF were followed prospectively for 5 years. Of these, 56 (53\%) developed recurrent paroxysmal AF and were placed on long-term antiarrhythmic drug therapy. AF became persistent in 24 of 45 patients taking antiarrhythmic drug therapy. In 11 such patients who failed antiarrhythmic drug therapy, RFA of AF was performed, and none of these patients had recurrence of AF. Among the persistent AF patients who failed drug therapy, 16 of 24 (67\%) progressed to permanent $\mathrm{AF}$, thus providing evidence for the superiority of RFA of AF in preventing progression of AF. Similarly, in the Ablation for Paroxysmal Atrial Fibrillation (APAF) trial, 198 patients with paroxysmal AF were randomly assigned to RFA or antiarrhythmic drug therapy. At 4 years of followup, by intention-to-treat analysis, $72.7 \%$ of patients in the ablation arm and $56.5 \%$ in the antiarrhythmic drug therapy arm remained free of recurrent $\mathrm{AF}(P=0.017) .{ }^{68}$ In this study, antiarrhythmic drug therapy in about $88 \%$ of patients was ineffective, requiring crossover to the RFA arm due to frequent recurrences and progression of AF. In another study by Jongnarangsin et al, ${ }^{23}$ a cohort of 504 patients with paroxysmal AF underwent RFA of AF and were followed over a mean duration of $27 \pm 12$ months. The progression rate to

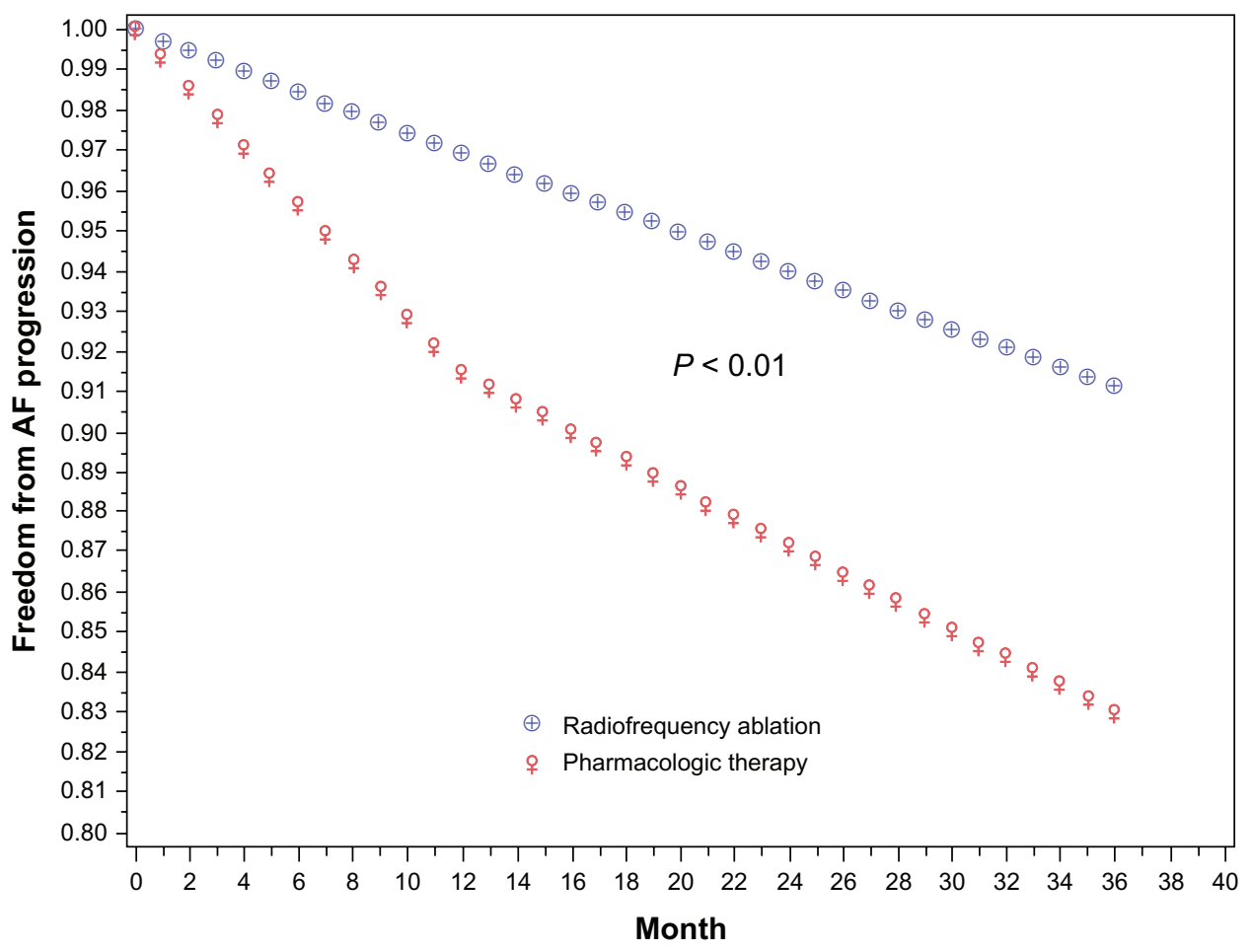

Figure 4 Rate of progression of atrial fibrillation. Probability for progression of atrial fibrillation was estimated to be $0.6 \%$ per year after radiofrequency catheter ablation (blue line) in comparison with a rate of $8.6 \%$ in the first year among patients treated with antiarrhythmic drug therapy. ${ }^{23}$

Note: Reprinted from Journal of Cardiovascular Electrophysiology, 23(I), Jongnarangsin K, Suwanagool A, Chugh A, et al, Effect of catheter ablation on progression of paroxysmal atrial fibrillation, 9-14, Copyright (C) 2012, with permission from John Wiley \& Sons.

Abbreviation: AF, atrial fibrillation. 


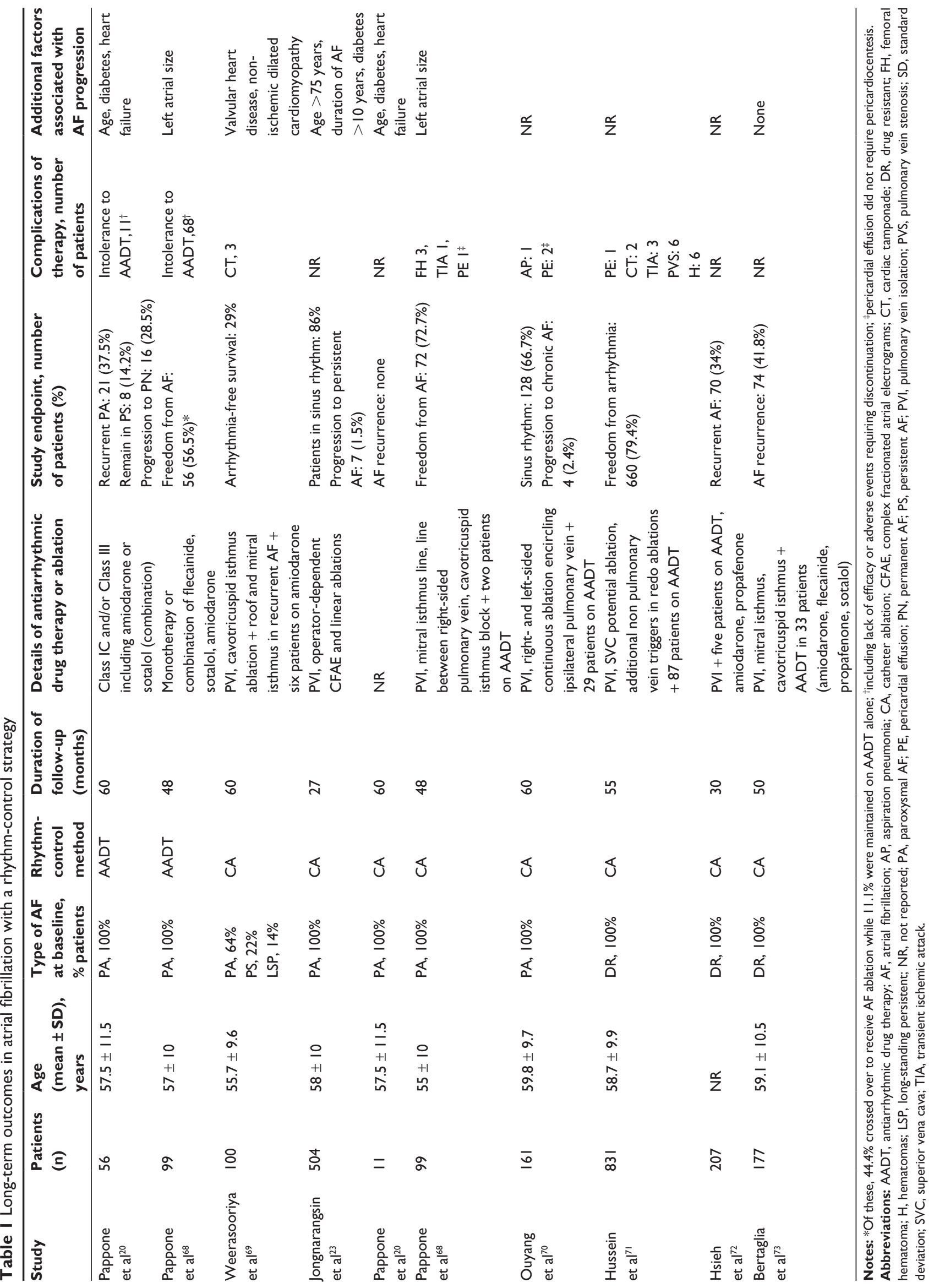


persistent AF was $0.6 \%$ per year after RFA in comparison with $9 \%$ per year as reported previously in pharmacologically treated patients (Figure 4 and Table 1).

The widespread acceptability of RFA as a first-line treatment modality for AF has been limited due to a lack of long-term follow-up data about the efficacy and safety profile across all patient groups, including those with underlying structural heart disease. Additionally, the procedure is invasive and is associated with a number of serious complications such as pulmonary vein stenosis, thromboembolism, atrio-esophageal fistula, and pericardial tamponade. Thus, it is important to understand which patients are less likely to benefit from ablation therapy. Until such questions are answered by well conducted randomized controlled trials, the true efficacy of catheter ablation across all patient populations and whether it just delays or truly prevents progression of AF remains to be proven conclusively.

\section{Conclusion}

$\mathrm{AF}$ is a progressive disease, and persistent or permanent AF is associated with greater patient morbidity and mortality than paroxysmal AF. Results from randomized controlled trials suggest no mortality benefit of a rhythm-control strategy for management of AF in comparison with a rate-control strategy. Nevertheless, use of a rate-control strategy is associated with more rapid progression of $\mathrm{AF}$ to persistent or permanent $\mathrm{AF}$, partly due to morphological changes in the atria that facilitate maintenance of AF. Thus, for younger or highly symptomatic patients with AF, the benefits associated with a rhythm-control strategy should especially be considered and implemented as appropriate. In select patients, catheter ablation provides greater success for prevention of progression of AF than antiarrhythmic drug therapy, although a need for an individualized approach for rhythm control remains essential.

\section{Disclosure}

Ashish Shukla reports no conflicts of interest in this work. Anne B Curtis discloses the following relationships: research grants: Medtronic (modest); honoraria: Medtronic (modest); consultant: Medtronic (modest); advisory board: SanofiAventis (modest), St Jude Medical (modest), Biosense Webster (modest), Janssen Pharmaceuticals (modest), Bristol-Myers-Squibb (modest).

\section{References}

1. Silverman ME. From rebellious palpitations to the discovery of auricular fibrillation: contributions of Mackenzie, Lewis and Einthoven. $\mathrm{Am} \mathrm{J}$ Cardiol. 1994;73(5):384-389.
2. Go AS, Hylek EM, Phillips KA, et al. Prevalence of diagnosed atrial fibrillation in adults: national implications for rhythm management and stroke prevention: the AnTicoagulation and Risk Factors in Atrial Fibrillation (ATRIA) Study. JAMA. 2001;285(18):2370-2375.

3. Miyasaka Y, Barnes ME, Gersh BJ, et al. Secular trends in incidence of atrial fibrillation in Olmsted County, Minnesota, 1980 to 2000, and implications on the projections for future prevalence. Circulation. 2006;114(2):119-125.

4. Sudlow M, Thomson R, Thwaites B, Rodgers H, Kenny RA. Prevalence of atrial fibrillation and eligibility for anticoagulants in the community. Lancet. 1998;352(9135):1167-1171.

5. Davis RC, Hobbs FD, Kenkre JE, et al. Prevalence of atrial fibrillation in the general population and in high-risk groups: the ECHOES study. Europace. 2012;14(11):1553-1559.

6. Lip GY, Brechin CM, Lane DA. The global burden of atrial fibrillation and stroke: a systematic review of the epidemiology of atrial fibrillation in regions outside North America and Europe. Chest. 2012;142(6):1489-1498.

7. Krahn AD, Manfreda J, Tate RB, Mathewson FA, Cuddy TE. The natural history of atrial fibrillation: incidence, risk factors, and prognosis in the Manitoba Follow-Up Study. Am J Med. 1995;98(5):476-484.

8. Lloyd-Jones DM, Wang TJ, Leip EP, et al. Lifetime risk for development of atrial fibrillation: the Framingham Heart Study. Circulation. 2004;110(9):1042-1046.

9. Heeringa J, van der Kuip DA, Hofman A, et al. Prevalence, incidence and lifetime risk of atrial fibrillation: the Rotterdam study. Eur Heart $J$. 2006;27(8):949-953.

10. Healey JS, Connolly SJ, Gold MR, et al. Subclinical atrial fibrillation and the risk of stroke. N Engl J Med. 2012;366(2):120-129.

11. Jabaudon D, Sztajzel J, Sievert K, Landis T, Sztajzel R. Usefulness of ambulatory 7-day ECG monitoring for the detection of atrial fibrillation and flutter after acute stroke and transient ischemic attack. Stroke. 2004;35(7):1647-1651.

12. Tayal AH, Tian M, Kelly KM, et al. Atrial fibrillation detected by mobile cardiac outpatient telemetry in cryptogenic TIA or stroke. Neurology. 2008;71(21):1696-1701.

13. Fuster V, Rydén LE, Cannom DS, et al; American College of Cardiology Foundation/American Heart Association Task Force. 2011 ACCF/ AHA/HRS focused updates incorporated into the ACC/AHA/ESC 2006 guidelines for the management of patients with atrial fibrillation: a report of the American College of Cardiology Foundation/American Heart Association Task Force on practice guidelines. Circulation. 2011;123(10):e269-e367.

14. Benjamin EJ, Chen PS, Bild DE, et al. Prevention of Atrial Fibrillation: Report From a National Heart, Lung, and Blood Institute workshop. Circulation. 2009;119(4):606-618.

15. Kerr CR, Humphries KH, Talajic M, et al. Progression to chronic atrial fibrillation after the initial diagnosis of paroxysmal atrial fibrillation: results from the Canadian Registry of Atrial Fibrillation. Am Heart J. 2005;149(3):489-496.

16. de Vos CB, Pisters R, Nieuwlaat R, et al. Progression from paroxysmal to persistent atrial fibrillation clinical correlates and prognosis. $J \mathrm{Am}$ Coll Cardiol. 2010;55(8):725-731.

17. De Vos CB, Breithardt G, Camm AJ, et al. Progression of atrial fibrillation in the REgistry on Cardiac rhythm disORDers assessing the control of Atrial Fibrillation cohort: clinical correlates and the effect of rhythm-control therapy. Am Heart J. 2012;163(5):887-893.

18. Nieuwlaat R, Prins MH, Le Heuzey JY, et al. Prognosis, disease progression, and treatment of atrial fibrillation patients during 1 year: follow-up of the Euro Heart Survey on atrial fibrillation. Eur Heart J. 2008;29(9):1181-1189.

19. Yamashita $T$, Inoue $H$, Okumura $K$, et al. Randomized trial of angiotensin II-receptor blocker vs dihydropiridine calcium channel blocker in the treatment of paroxysmal atrial fibrillation with hypertension (J-RHYTHM II study). Europace. 2011;13(4): 473-479. 
20. Pappone C, Radinovic A, Manguso F, et al. Atrial fibrillation progression and management: a 5-year prospective follow-up study. Heart Rhythm. 2008;5(11):1501-1507.

21. Tsang TS, Barnes ME, Miyasaka Y, et al. Obesity as a risk factor for the progression of paroxysmal to permanent atrial fibrillation: a longitudinal cohort study of 21 years. Eur Heart J. 2008;29(18):2227-2233.

22. Le Heuzey JY, Breithardt G, Camm J, et al. The RecordAF study: design, baseline data, and profile of patients according to chosen treatment strategy for atrial fibrillation. Am J Cardiol. 2010;105(5):687-693.

23. Jongnarangsin K, Suwanagool A, Chugh A, et al. Effect of catheter ablation on progression of paroxysmal atrial fibrillation. J Cardiovasc Electrophysiol. 2012;23(1):9-14.

24. Cooper HA, Bloomfield DA, Bush DE, et al. Relation between achieved heart rate and outcomes in patients with atrial fibrillation (from the Atrial Fibrillation Follow-up Investigation of Rhythm Management [AFFIRM] Study). Am J Cardiol. 2004;93(10):1247-1253.

25. American College of Cardiology Foundation, American Heart Association, European Society of Cardiology, Heart Rhythm Society, et al. Management of patients with atrial fibrillation (compilation of $2006 \mathrm{ACCF} / \mathrm{AHA} / \mathrm{ESC}$ and $2011 \mathrm{ACCF} / \mathrm{AHA} / \mathrm{HRS}$ recommendations): a report of the American College of Cardiology/American Heart Association Task Force on practice guidelines. Circulation. 2013;127(18): 1916-1926.

26. Groenveld HF, Tijssen JG, Crijns HJ, et al. Rate control efficacy in permanent atrial fibrillation: successful and failed strict rate control against a background of lenient rate control: data from RACE II (Rate Control Efficacy in Permanent Atrial Fibrillation). J Am Coll Cardiol. 2013;61(7):741-748.

27. Burashnikov A, Di Diego JM, Zygmunt AC, Belardinelli L, Antzelevitch C. Atrium-selective sodium channel block as a strategy for suppression of atrial fibrillation: differences in sodium channel inactivation between atria and ventricles and the role of ranolazine. Circulation. 2007;116(13):1449-1457.

28. Zimetbaum P. Antiarrhythmic drug therapy for atrial fibrillation. Circulation. 2012;125(2):381-389.

29. Sokolow M. Quinidine in the treatment of benign auricular fibrillation with repeated emboli: report of a case. Am Heart J. 1939;18(4): 494-499.

30. Södermark T, Jonsson B, Olsson A, et al. Effect of quinidine on maintaining sinus rhythm after conversion of atrial fibrillation or flutter. A multicentre study from Stockholm. Br Heart J. 1975;37(5):486-492.

31. Lloyd EA, Gersh BJ, Forman R. The efficacy of quinidine and disopyramide in the maintenance of sinus rhythm after electroconversion from atrial fibrillation. A double-blind study comparing quinidine, disopyramide and placebo. S Afr Med J. 1984;65(10):367-369.

32. Karlson BW, Torstensson I, Abjörn C, Jansson SO, Peterson LE. Disopyramide in the maintenance of sinus rhythm after electroconversion of atrial fibrillation. A placebo-controlled one-year follow-up study. Eur Heart J. 1988;9(3):284-290.

33. Crijns HJ, Gosselink AT, Lie KI. Propafenone versus disopyramide for maintenance of sinus rhythm after electrical cardioversion of chronic atrial fibrillation: a randomized, double-blind study. PRODIS Study Group. Cardiovasc Drugs Ther. 1996;10(2):145-152.

34. Aliot E, Denjoy I. Comparison of the safety and efficacy of flecainide versus propafenone in hospital out-patients with symptomatic paroxysmal atrial fibrillation/flutter. The Flecainide AF French Study Group. Am J Cardiol. 1996;77(3):66A-71A.

35. Chimienti M, Cullen MT Jr, Casadei G. Safety of long-term flecainide and propafenone in the management of patients with symptomatic paroxysmal atrial fibrillation: report from the Flecainide and Propafenone Italian Study Investigators. Am J Cardiol. 1996;77(3):60A-75A.

36. Roy D, Talajic M, Dorian P, et al. Amiodarone to prevent recurrence of atrial fibrillation. Canadian Trial of Atrial Fibrillation Investigators. $N$ Engl J Med. 2000;342(13):913-920.

37. Singh BN, Singh SN, Reda DJ, et al. Amiodarone versus sotalol for atrial fibrillation. $N$ Engl J Med. 2005;352(18):1861-1872.
38. AFFIRM First Antiarrhythmic Drug Substudy Investigators. Maintenance of sinus rhythm in patients with atrial fibrillation: an AFFIRM substudy of the first antiarrhythmic drug. J Am Coll Cardiol. 2003;42(1): 20-29.

39. Freemantle N, Lafuente-Lafuente C, Mitchell S, Eckert L, Reynolds M. Mixed treatment comparison of dronedarone, amiodarone, sotalol, flecainide, and propafenone, for the management of atrial fibrillation. Europace. 2011;13(3):329-345.

40. Lafuente-Lafuente C, Mouly S, Longás-Tejero MA, Mahé I, Bergmann JF. Antiarrhythmic drugs for maintaining sinus rhythm after cardioversion of atrial fibrillation: a systematic review of randomized controlled trials. Arch Intern Med. 2006;166(7):719-728.

41. Vassallo P, Trohman RG. Prescribing amiodarone: an evidence-based review of clinical indications. JAMA. 2007;298(11):1312-1322.

42. Singh BN, Connolly SJ, Crijns HJ, et al. Dronedarone for maintenance of sinus rhythm in atrial fibrillation or flutter. N Engl J Med. 2007;357(10): 987-999.

43. Hohnloser SH, Crijns HJ, van Eickels M, et al; ATHENA Investigators. Effect of dronedarone on cardiovascular events in atrial fibrillation. N Engl J Med. 2009;360(7):668-678.

44. Køber L, Torp-Pedersen C, McMurray JJ, et al. Increased mortality after dronedarone therapy for severe heart failure. NEngl JMed.2008;358(25): 2678-2687.

45. Connolly SJ, Camm AJ, Halperin JL, et al. Dronedarone in high-risk permanent atrial fibrillation. N Engl J Med. 2011;365(24):2268-2276.

46. Curtis AB. Practice Implications of the Atrial Fibrillation Guidelines. Am J Cardiol. 2013;111(11):1660-1670.

47. Anderson JL, Prystowsky EN. Sotalol: an important new antiarrhythmic Am Heart J. 1999;137(3):388-409.

48. Singh S, Zoble RG, Yellen L, et al. Efficacy and safety of oral dofetilide in converting to and maintaining sinus rhythm in patients with chronic atrial fibrillation or atrial flutter: the symptomatic atrial fibrillation investigative research on dofetilide (SAFIRE-D) study. Circulation. 2000;102(19):2385-2390.

49. Pedersen OD, Bagger H, Keller N, Marchant B, Køber L, Torp-Pedersen C. Efficacy of dofetilide in the treatment of atrial fibrillation-flutter in patients with reduced left ventricular function: a Danish investigations of arrhythmia and mortality on dofetilide (diamond) substudy. Circulation. 2001;104(3):292-296.

50. Oral H, Souza JJ, Michaud GF, et al. Facilitating transthoracic cardioversion of atrial fibrillation with ibutilide pretreatment. $N$ Engl J Med. 1999;340(24):1849-1854.

51. Hongo RH, Themistoclakis S, Raviele A, et al. Use of ibutilide in cardioversion of patients with atrial fibrillation or atrial flutter treated with class IC agents. J Am Coll Cardiol. 2004;44(4):864-868.

52. Chiladakis JA, Kalogeropoulos A, Patsouras N, Manolis AS. Ibutilide added to propafenone for the conversion of atrial fibrillation and atrial flutter. J Am Coll Cardiol. 2004;44(4):859-863.

53. Camm AJ, Lip GY, De Caterina R, et al. 2012 focused update of the ESC Guidelines for the management of atrial fibrillation: an update of the 2010 ESC Guidelines for the management of atrial fibrillation. Developed with the special contribution of the European Heart Rhythm Association. Eur Heart J. 2012;33(21):2719-2747.

54. Calkins H. Catheter ablation to maintain sinus rhythm. Circulation. 2012;125(11):1439-1445.

55. Wazni OM, Marrouche NF, Martin DO, et al. Radiofrequency ablation vs antiarrhythmic drugs as first-line treatment of symptomatic atrial fibrillation: a randomized trial. JAMA. 2005;293(21):2634-2640.

56. Stabile G, Bertaglia E, Senatore G, et al. Catheter ablation treatment in patients with drug-refractory atrial fibrillation: a prospective, multicentre, randomized, controlled study (Catheter Ablation For The Cure Of Atrial Fibrillation Study). Eur Heart J. 2006;27(2):216-221.

57. Pappone C, Augello G, Sala S, et al. A randomized trial of circumferential pulmonary vein ablation versus antiarrhythmic drug therapy in paroxysmal atrial fibrillation: the APAF Study. J Am Coll Cardiol. 2006;48(11):2340-2347. 
58. Noheria A, Kumar A, Wylie JV Jr, Josephson ME. Catheter ablation vs antiarrhythmic drug therapy for atrial fibrillation: a systematic review. Arch Intern Med. 2008;168(6):581-586.

59. Cosedis Nielsen J, Johannessen A, Raatikainen P, et al. Radiofrequency ablation as initial therapy in paroxysmal atrial fibrillation. $N$ Engl $J$ Med. 2012;367(17):1587-1595.

60. Thacker EL, McKnight B, Psaty BM, et al. Association of body mass index, diabetes, hypertension, and blood pressure levels with risk of permanent atrial fibrillation. J Gen Intern Med. 2013;28(2): 247-253.

61. Wang TJ, Parise H, Levy D, et al. Obesity and the risk of new-onset atrial fibrillation. JAMA. 2004;292(20):2471-2477.

62. Bitter T, Nölker G, Vogt J, Prinz C, Horstkotte D, Oldenburg O. Predictors of recurrence in patients undergoing cryoballoon ablation for treatment of atrial fibrillation: the independent role of sleep-disordered breathing. J Cardiovasc Electrophysiol. 2012;23(1):18-25.

63. Patel D, Mohanty P, Di Biase L, et al. Safety and efficacy of pulmonary vein antral isolation in patients with obstructive sleep apnea: the impact of continuous positive airway pressure. Circ Arrhythm Electrophysiol. 2010;3(5):445-451.

64. Camm AJ, Breithardt G, Crijns H, et al. Real-life observations of clinical outcomes with rhythm- and rate-control therapies for atrial fibrillation RECORDAF (Registry on Cardiac Rhythm Disorders Assessing the Control of Atrial Fibrillation). J Am Coll Cardiol. 2011;58(5): 493-501.

65. Lu Z, Scherlag BJ, Lin J, et al. Atrial fibrillation begets atrial fibrillation: autonomic mechanism for atrial electrical remodeling induced by short-term rapid atrial pacing. Circ Arrhythm Electrophysiol. 2008;1(3): 184-192.
66. Hagens VE, Van Veldhuisen DJ, Kamp O, et al. Effect of rate and rhythm control on left ventricular function and cardiac dimensions in patients with persistent atrial fibrillation: results from the RAte Control versus Electrical Cardioversion for Persistent Atrial Fibrillation (RACE) study. Heart Rhythm. 2005;2(1):19-24.

67. Calkins H, Reynolds MR, Spector P, et al. Treatment of atrial fibrillation with antiarrhythmic drugs or radiofrequency ablation: two systematic literature reviews and meta-analyses. Circ Arrhythm Electrophysiol. 2009;2(4):349-361.

68. Pappone C, Vicedomini G, Augello G, et al. Radiofrequency catheter ablation and antiarrhythmic drug therapy: a prospective, randomized, 4-year follow-up trial: the APAF study. Circ Arrhythm Electrophysiol. 2011;4(6):808-814.

69. Weerasooriya R, Khairy P, Litalien J, et al. Catheter ablation for atrial fibrillation: are results maintained at 5 years of follow-up? J Am Coll Cardiol. 2011;57(2):160-166.

70. Ouyang F, Tilz R, Chun J, et al. Long-term results of catheter ablation in paroxysmal atrial fibrillation: lessons from a 5-year follow-up. Circulation. 2010;122(23):2368-2377.

71. Hussein AA, Saliba WI, Martin DO, et al. Natural history and long-term outcomes of ablated atrial fibrillation. Circ Arrhythm Electrophysiol. 2011;4(3):271-278

72. Hsieh MH, Tai CT, Tsai CF, et al. Clinical outcome of very late recurrence of atrial fibrillation after catheter ablation of paroxysmal atrial fibrillation. J Cardiovasc Electrophysiol. 2003;14(6):598-601.

73. Bertaglia E, Tondo C, De Simone A, et al. Does catheter ablation cure atrial fibrillation? Single-procedure outcome of drug-refractory atrial fibrillation ablation: a 6-year multicentre experience. Europace. 2010;12(2):181-187.
Vascular Health and Risk Management

\section{Publish your work in this journal}

Vascular Health and Risk Management is an international, peerreviewed journal of therapeutics and risk management, focusing on concise rapid reporting of clinical studies on the processes involved in the maintenance of vascular health; the monitoring, prevention and treatment of vascular disease and its sequelae; and the involvement of

\section{Dovepress}

metabolic disorders, particularly diabetes. This journal is indexed on PubMed Central and MedLine. The manuscript management system is completely online and includes a very quick and fair peer-review system, which is all easy to use. Visit http://www.dovepress.com/ testimonials.php to read real quotes from published authors. 